\title{
LARGE-ORDER ASYMPTOTES FOR DYNAMIC MODELS NEAR EQUILIBRIUM
}

\author{
Juha Honkonen $^{\mathrm{a}, *}$, M. V. Komarova ${ }^{\mathrm{b}}$ and M. Yu. Nalimov ${ }^{\mathrm{b}}$ \\ a Theoretical Physics Division, Department of Physical Sciences, \\ P.O. Box 64,FIN-00014 University of Helsinki, Finland \\ b Department of Theoretical Physics, St. Petersburg University, Uljanovskaja 1, \\ St. Petersburg, Petrodvorets, 198504 Russia
}

\begin{abstract}
Instanton analysis is applied to model A of critical dynamics. It is shown that the static instanton of the massless $\phi^{4}$ model determines the large-order asymptotes of the perturbation expansion of the dynamic model.
\end{abstract}

Key words: instanton, dynamic models, large orders

PACS: 11.10.Jj, 05.70.Jk

\section{Introduction}

The knowledge of large-order asymptotic behaviour of perturbation series of static field-theoretic models is important for resummation of series for critical exponents and scaling functions [1]. This behaviour has been thoroughly explored with the aid of instanton analysis and applied to the resummation problem in the prototypical static $\phi^{4}$ model $[2,3,4]$, which has been widely used as a model of critical behaviour in continuous phase transitions of ferromagnetic type.

\footnotetext{
* corresponding author

Email addresses: Juha.Honkonen@helsinki.fi (Juha Honkonen), komarova@paloma.spbu.ru (M. V. Komarova),

Mikhail.Nalimov@pobox.spbu.ru (M. Yu. Nalimov).
} 
However, little is known about large-order asymptotics in dynamic field theories constructed from Langevin equations with the aid of the Martin-SiggiaRose (MSR) formalism [5]. Recently, it was stated [6,7] that there is no instanton within the MSR approach in the Kraichnan model - which has attracted considerable attention as a model describing intermittency in turbulent diffusion [8] - and that the method of steepest descent has to be used in Lagrangean variables.

In this paper we propose a method to assess large-order asymptotic behaviour of dynamic models near equilibrium, i.e. with Gibbsian static limit (we also will restrict ourselves to models generated by Langevin equations without mode coupling). We will use the steepest descent method (instanton approach) to find the large-order behaviour in a representative model. We will discuss large-order asymptotes of correlation functions, response functions and critical exponents. For equal-time correlation functions this asymptotic behaviour is shown to coincide with that of the static instanton approach.

The particular model we deal with in this paper is one of the standard dynamic $\phi^{4}$-based models: model A in the classification of Ref. [9]. In this model critical exponents are the same as in the static $\phi^{4}$ model apart from the dynamic exponent $z$. The large-order asymptotics of the dynamic exponent have not been analyzed so far. It should be noted here that the use of Lagrangean variables becomes prohibitively difficult in this case due to the essential nonlinearity of the problem (the Kraichnan model is linear in the advected scalar field).

The present article is organized as follows: in Sec. 2 construction of the MSR field theory corresponding to a non-linear Langevin equation is reviewed with special emphasis on the treatment of the functional determinant for the steepest descent method. Existence of the dynamic instanton and relation of the dynamic instanton solution to the static one is analysed in Sec. 3. The fluctuation determinant is calculated in Sec. 4, whereas Sec. 5 is devoted to a brief analysis of correlation and response functions. Results of this paper are summarized in Sec. 6.

\section{Dynamic field theory}

Consider the Langevin equation

$$
\frac{\partial \varphi}{\partial t}+\Gamma \frac{\delta S}{\delta \varphi}=\xi
$$


where $\xi$ is a Gaussian random field with zero mean and the correlation function

$$
\left\langle\xi(t, \mathbf{x}) \xi\left(t^{\prime}, \mathbf{x}^{\prime}\right)\right\rangle=D\left(x-x^{\prime}\right)=2 \Gamma \delta\left(t-t^{\prime}\right) \delta\left(\mathbf{x}-\mathbf{x}^{\prime}\right),
$$

where the shorthand notation $x=(t, \mathbf{x})$ has been used. In equation (1) the action is the static action of arbitrary model with the known instanton. The most interesting example is the massless $\varphi^{4}$ model:

$$
S=\frac{1}{2} \partial \varphi \partial \varphi+\frac{g}{4 !} \varphi^{4} .
$$

Here and henceforth, all necessary integrals and sums are implied. We find it convenient to start the analysis on a finite time interval $\left[t_{0}, T\right]$, but in the full Euclidian space $\mathbf{R}^{D}$. In the MSR approach, functional integrals for correlation and response functions are calculated with the "measure"

$$
\mathfrak{M}\left[\varphi, \varphi^{\prime}\right]=\mathcal{D} \varphi \mathcal{D} \varphi^{\prime} \operatorname{det} M e^{-\bar{S}+A_{\varphi} \varphi+A_{\varphi^{\prime}} \varphi^{\prime}},
$$

where the dynamic action is of the De Dominicis-Janssen form [10]

$$
\bar{S}=-\frac{1}{2} \varphi^{\prime} D \varphi^{\prime}+\varphi^{\prime}\left(\frac{\partial \varphi}{\partial t}+\Gamma \frac{\delta S}{\delta \varphi}\right) .
$$

The operator determinant $\operatorname{det} M$ in (3) has different interpretations. Most often it is written as a result of a formal change of variables in a $\delta$ functional imposing condition (1), which yields $[1,10]$

$$
M=\frac{\partial \delta\left(x-x^{\prime}\right)}{\partial t}+\Gamma \frac{\delta^{2} S}{\delta \varphi(x) \delta \varphi\left(x^{\prime}\right)}
$$

for the operator $M$, after which the determinant is calculated in a formal loop expansion as

$$
\operatorname{det} M=\operatorname{det}\left[\left(\frac{\partial}{\partial t}+\Gamma K\right) \delta\left(x-x^{\prime}\right)\right] \exp \left[\frac{1}{2} \iint d x d x^{\prime} \frac{\delta^{2} S_{I}}{\delta \varphi(x) \delta \varphi\left(x^{\prime}\right)}\right]
$$

where $K$ is the differential operator of the free-field part of the static action [e.g. $K=-\nabla^{2}$ for action (2)] and $S_{I}$ its interaction part. An annoying feature of this straightforward method is that the normalization determinant $\operatorname{det}\left[\left(\partial_{t}+\Gamma K\right) \delta\left(x-x^{\prime}\right)\right]=\operatorname{det} M_{0}$ is that of a non-self-adjoint operator. The explicit content of this factor is unimportant in the ordinary perturbation theory, because in the calculation of all expectation values it simply cancels out in each term separately. However, in instanton calculus the saddle-point solution which serves as the expansion point for a steepest-descent calculation of the functional integral is different from that of the ordinary perturbation expansion. Therefore, the possible cancellation of determinants requires additional analysis, which is much more convenient to carry out in terms of determinants of self-adjoint operators. 
It is quite possible and completely consistent to arrive at a functional measure, in which the normalizing determinant is that of a self-adjoint operator. To this end, recall the basics of the MSR construction, in which classical quantities are replaced by field operators $\hat{\psi}, \hat{\psi}^{\prime}$ with the usual bosonic commutation relations $\left\{\right.$ normalized as $\left.\left[\hat{\psi}(t, \mathbf{x}), \hat{\psi}^{\prime}\left(t, \mathbf{x}^{\prime}\right)\right]=\delta\left(\mathbf{x}-\mathbf{x}^{\prime}\right)\right\}$ and the dynamics are given by the operator equations [5]

$$
\frac{\partial \hat{\psi}}{\partial t}=[\hat{\psi}, \hat{\mathcal{H}}], \quad \frac{\partial \hat{\psi}^{\prime}}{\partial t}=\left[\hat{\psi}^{\prime}, \hat{\mathcal{H}}\right]
$$

where the MSR "Hamiltonian" is

$$
\hat{\mathcal{H}}=-\frac{1}{2} \hat{\psi}^{\prime} D \hat{\psi}^{\prime}+\left.\Gamma \hat{\psi}^{\prime}\left[\frac{\delta S(\varphi)}{\delta \varphi}\right]\right|_{\varphi \rightarrow \hat{\psi}}
$$

We have chosen the MSR Hamiltonian in the form (6), which is slightly different from the originally proposed (although this possibility was discussed in the original paper [5]), because we want to write the free-field part of the dynamic action in the form of a formally convergent Gaussian integral at the outset. The chosen MSR Hamiltonian corresponds to the stage, where all integrations over the random source $\xi$ of Langevin equation (1) have been carried out, and - integrated over time - it yields the dynamic action (4).

With the use of the traditional operator formalism the generating functional of Green functions of these fields may be expressed in the functional form [11]

$$
G\left(A_{\varphi}, A_{\varphi^{\prime}}\right)=\left.\exp \left[\frac{1}{2} \frac{\delta}{\delta \Psi} \Delta \frac{\delta}{\delta \Psi}\right] \exp \left[\bar{S}_{I}+A_{\varphi} \psi+A_{\varphi^{\prime}} \psi^{\prime}\right]\right|_{\Psi=0}
$$

where $\bar{S}_{I}$ is the time integral of the interaction part of the MSR Hamiltonian (6) in the interaction picture, whereas the kernel of the functional differential operator - written in terms of the two-component field $\Psi=\left(\psi, \psi^{\prime}\right)$ - is the propagator

$$
\begin{gathered}
\Delta\left(x, x^{\prime}\right)=\left[\begin{array}{cc}
\Delta_{11}\left(x, x^{\prime}\right) & \Delta_{12}\left(x, x^{\prime}\right) \\
\Delta_{21}\left(x, x^{\prime}\right) & 0
\end{array}\right] \\
=\left\{\begin{array}{c}
{\left[\left(\partial_{t}+\Gamma K\right)^{-1} D\left(\partial_{t}+\Gamma K\right)^{-T}\right]\left(x, x^{\prime}\right)\left(\partial_{t}+\Gamma K\right)^{-1}\left(x, x^{\prime}\right)} \\
\left(\partial_{t}+\Gamma K\right)^{-T}\left(x, x^{\prime}\right)
\end{array}\right\} \\
=\left\{\begin{array}{c}
{\left[M_{0}^{-1} D M_{0}^{-T}\right]\left(x, x^{\prime}\right) M_{0}^{-1}\left(x, x^{\prime}\right)} \\
M_{0}^{-T}\left(x, x^{\prime}\right)
\end{array}\right] .
\end{gathered}
$$

In this approach the functional integral arises from expressing the functional 
differential operator in (7) as the Gaussian functional integral:

$$
\begin{aligned}
& \exp \left[\frac{1}{2} \frac{\delta}{\delta \Psi} \Delta \frac{\delta}{\delta \Psi}\right]=\sqrt{\operatorname{det} M_{0}^{T} M_{0}} \\
& \times \int \mathcal{D} \varphi \mathcal{D} \varphi^{\prime} \exp \left[-\frac{1}{2} \Phi \mathcal{K} \Phi+\varphi \frac{\delta}{\delta \psi}+\varphi^{\prime} \frac{\delta}{\delta \psi^{\prime}}\right]
\end{aligned}
$$

where $\Phi=\left(\varphi, \varphi^{\prime}\right)$ and the operator $\mathcal{K}$ satisfies $\mathcal{K} \Delta=1$ with the appropriate boundary conditions. This is the only place where a functional determinant arises and now it is that of a self-adjoint operator $M_{0}^{T} M_{0}$ completely determined by the free-field part of the MSR Hamiltonian.

It might be asked what happened to the field-dependent extra factor in (5)? The answer depends on the definition of the time-ordered product ( $T$ product) at coinciding time arguments. If the time-ordered product at equal times is defined as the symmetric product of the field operators, then the interaction functional $\bar{S}_{I}$ in (7) is the Sym form [11] of the interaction part of the MSR Hamiltonian. The Sym form of the MSR Hamiltonian (6) contains terms quadratic in fields which exactly correspond to the field-dependent factor in (5). It is quite possible, and here in fact more convenient, to define the coinciding-time time-ordered product as the normal product [11] of the field operators. In this case the interaction functional $\bar{S}_{I}$ is taken in the normal form, which in the perturbation theory implies the condition that there are no graphs with closed loops of the retarded propagator $\left(\partial_{t}+\Gamma K\right)^{-1}\left(x, x^{\prime}\right)$ attached to the interaction vertex. This amounts to vanishing retarded free-field propagator at coinciding times, and no additional factors to the determinant $\operatorname{det} M_{0}^{T} M_{0}$ are generated. Henceforth, we will use the latter approach which allows to choose the determinant factor of (3) in the field-independent form $\operatorname{det} M=\sqrt{\operatorname{det} M_{0}^{T} M_{0}}$.

In the instanton approach the space of integration in the functional integral is chosen using the properties of the saddle point and may well be different from that of the ordinary perturbation theory. Since the starting point is the functional integral constructed for perturbation theory, let us recall what the appropriate space of integration is in that case. In the proper choice of the formal space of integration there are two main points to be taken into account [11]: the operator $\mathcal{K}$ must be nondegenerate and the kernel of the uniquely defined operator $\mathcal{K}^{-1}$ must be the propagator $\Delta$ used for the construction of the perturbation theory. For the model generated by the static action (2) the usual diffusion kernel in unbounded space

$$
\left(\partial_{t}-\Gamma \nabla^{2}\right)^{-1}\left(x, x^{\prime}\right)=\frac{\theta\left(t-t^{\prime}\right) \exp \left[-\frac{\left(\mathbf{x}-\mathbf{x}^{\prime}\right)^{2}}{4 \Gamma\left(t-t^{\prime}\right)}\right]}{\left[4 \pi \Gamma\left(t-t^{\prime}\right)\right]^{d / 2}}
$$


is the choice to generate the propagator $\Delta$ in (8). The space of integration $E(\Delta)$ conforming to these requirements may be constructed as

$$
\left(\begin{array}{c}
\varphi \\
\varphi^{\prime}
\end{array}\right)=\left(\begin{array}{cc}
\Delta_{11} & \Delta_{12} \\
\Delta_{21} & 0
\end{array}\right)\left(\begin{array}{c}
\eta \\
\eta^{\prime}
\end{array}\right)=\left(\begin{array}{l}
\Delta_{11} \eta+\Delta_{12} \eta^{\prime} \\
\Delta_{21} \eta
\end{array}\right),
$$

where $\eta$ and $\eta^{\prime}$ are fields vanishing at the boundaries (i.e. at $|\mathbf{x}| \rightarrow \infty, t=t_{0}$ and $t=T$ ) to ensure all partial integrations in the differential operators of $\mathcal{K}$ without surface terms. It is worth noting that $\varphi^{\prime}(T)=0$, but both fields have finite (and real-valued) initial values at $t=t_{0}$.

In terms of fields vanishing at the boundaries the right-hand side of equation (9) assumes a bit different form giving rise to the representation

$$
\begin{array}{r}
\exp \left[\frac{1}{2} \frac{\delta}{\delta \Psi} \Delta \frac{\delta}{\delta \Psi}\right]=\sqrt{\operatorname{det} \Delta_{12} \Delta_{21}} \int \mathcal{D} \eta^{\prime} \mathcal{D} \eta \exp \left[-\frac{1}{2} \eta \Delta_{11} \eta-\eta \Delta_{12} \eta^{\prime}\right] \\
\times \exp \left[\left(\Delta_{11} \eta+\Delta_{12} \eta^{\prime}\right) \frac{\delta}{\delta \psi}+\Delta_{21} \eta \frac{\delta}{\delta \psi^{\prime}}\right]
\end{array}
$$

in which the Gaussian functional integral is taken over fields vanishing at boundaries. Nevertheless, formal convergence requires it to be understood as an iterated integral in the order indicated in (11), the field $\eta$ to be real and the field $\eta^{\prime}$ purely imaginary.

\section{Instanton analysis}

Let us calculate the parametric integral expressing the $N$ th order contribution to perturbation expansion in $g$ of the $k$-point correlation function

$$
\frac{1}{2 \pi i} \oint \frac{d g}{g} \frac{\iint \mathcal{D} \varphi \mathcal{D} \varphi^{\prime} \varphi\left(\mathbf{x}_{\mathbf{1}}, t_{1}\right) \ldots \varphi\left(\mathbf{x}_{\mathbf{k}}, t_{k}\right) e^{-\bar{S}-N \lg g}}{\iint \mathcal{D} \varphi \mathcal{D} \varphi^{\prime} e^{-\bar{S}_{0}}}
$$

by the method of steepest descent in the variables $g$ and $\varphi$. We have written the normalizing determinant in the form of the functional integral

$$
\left(\operatorname{det} M_{0}^{T} M_{0}\right)^{-1 / 2}=\iint \mathcal{D} \varphi \mathcal{D} \varphi^{\prime} e^{-\bar{S}_{0}}
$$

with the free-field part of the dynamic action

$$
\bar{S}_{0}=-\frac{1}{2} \varphi^{\prime} D \varphi^{\prime}+\varphi^{\prime}\left(\frac{\partial \varphi}{\partial t}+\Gamma K \varphi\right)
$$


in order to make explicit the cancellation of Jacobians accompanying several forthcoming changes of variables. We will show that the instanton solution for expression (12) may be constructed in close relation to the instanton solution of the corresponding equilibrium model. Therefore, it is worth reminding that the equilibrium expression for the $k$-point correlation function without vacuum loops [there are no vacuum loops in the dynamic correlation function (12)] of the field $\varphi$ is recovered in the limit $t_{0} \rightarrow-\infty$ for $t_{1}=t_{2}=\ldots=t_{k}$.

Applying the instanton approach we make - in analogy with the usual instanton analysis of the $\varphi^{4}$ model - the change of variables $\varphi \rightarrow \sqrt{N} \varphi, \varphi^{\prime} \rightarrow \sqrt{N} \varphi^{\prime}$, $g \rightarrow g / N$; in case of any other model it is convenient to scale the field variables extracting the dependence of $N$ in the expression for $\bar{S}$. The corresponding Jacobians from the numerator and the denominator of (12) cancel. The stationarity equations for the exponential of the numerator are

$$
\begin{aligned}
& \frac{\delta \bar{S}}{\delta \varphi}=-\frac{\partial \varphi^{\prime}}{\partial t}+\Gamma \frac{\delta^{2} S}{\delta \varphi^{2}} \varphi^{\prime}=0 \\
& \frac{\delta \bar{S}}{\delta \varphi^{\prime}}=-D \varphi^{\prime}+\frac{\partial \varphi}{\partial t}+\Gamma \frac{\delta S}{\delta \varphi}=0 \\
& \frac{\partial \bar{S}}{\partial g}=-\frac{1}{g}
\end{aligned}
$$

To construct the instanton solution, consider the following auxiliary equation [note the change of sign of the time derivative in comparison with the the Langevin equation (1) and the corresponding stationarity equation (14)]

$$
-\frac{\partial \varphi}{\partial t}+\Gamma \frac{\delta S}{\delta \varphi}=0
$$

and assume for the time being that there is a solution $\varphi_{d}$ conforming the Cauchy condition for the final time instant $\varphi_{d}(T, \mathbf{x})=\varphi_{f}(\mathbf{x})$ with a sufficiently smooth function $\varphi_{f}(\mathbf{x})$. Substitution of this solution to equation (14) yields

$$
-D \varphi^{\prime}+\frac{\partial \varphi_{d}}{\partial t}+\left.\Gamma \frac{\delta S}{\delta \varphi}\right|_{\varphi \rightarrow \varphi_{d}}=-D \varphi^{\prime}+2 \frac{\partial \varphi_{d}}{\partial t}=0
$$

which leads to the nontrivial $\varphi_{d}^{\prime}$

$$
\varphi_{d}^{\prime}=2 D^{-1} \frac{\partial \varphi_{d}}{\partial t}
$$

On the other hand, equation (13) on this solution is satisfied automatically: indeed, taking the time derivative of equation (16) yields

$$
\frac{\partial}{\partial t} \frac{\partial \varphi_{d}}{\partial t}-\left.\Gamma \frac{\delta^{2} S}{\delta \varphi \delta \varphi}\right|_{\varphi \rightarrow \varphi_{d}} \frac{\partial \varphi_{d}}{\partial t}=\left(\frac{\partial}{\partial t}-\left.\Gamma \frac{\delta^{2} S}{\delta \varphi \delta \varphi}\right|_{\varphi \rightarrow \varphi_{d}}\right) \frac{\partial \varphi_{d}}{\partial t}=0
$$


which, apart from a multiplicative factor, is equation (13) on the solution (17).

Finally, equation (15) imposes an asymptotic condition on $\varphi_{d}$ : it may be cast in the form (we remind that on the left-hand side of this equation integrations over time and space in the action are implied and intact)

$$
\begin{aligned}
\frac{\partial \bar{S}}{\partial g}=\left.\Gamma \varphi_{d}^{\prime} \frac{\partial}{\partial g} \frac{\delta S}{\delta \varphi}\right|_{\varphi \rightarrow \varphi_{d}}=\left.\int_{t_{0}}^{T} d t \frac{d}{d t} \frac{\partial S}{\partial g}\right|_{\varphi \rightarrow \varphi_{d}} & \\
& =\lim _{t \rightarrow T} \frac{\partial S\left(\varphi_{d}\right)}{\partial g}-\lim _{t \rightarrow t_{0}} \frac{\partial S\left(\varphi_{d}\right)}{\partial g}=-\frac{1}{g}
\end{aligned}
$$

since $\Gamma \varphi_{d}^{\prime}=2 \Gamma D^{-1} \partial_{t} \varphi_{d}=\partial_{t} \varphi_{d}$. Taking into account the boundary conditions imposed on the solution of equation (16) we immediately see that on the solution (16) and (17) the third saddle-point equation (15) reduces to the following equation for the limiting values of the dynamic instanton

$$
\frac{\partial S\left(\varphi_{f}\right)}{\partial g}-\frac{\partial S\left(\varphi_{0}\right)}{\partial g}=-\frac{1}{g}
$$

of which $\varphi_{f}$ is the condition for the Cauchy problem and $\varphi_{0}(\mathbf{x})=\varphi_{d}\left(t_{0}, \mathbf{x}\right)$. Since equation (16) gives rise to iterative solution with the advanced diffusion kernel, we see that sufficiently small "initial" field $\varphi_{0}$ vanishes in the limit $t_{0} \rightarrow-\infty$ and we thus recover the usual equation for the static instanton

$$
\frac{\partial S\left(\varphi_{f}\right)}{\partial g}=-\frac{1}{g}, \quad t_{0} \rightarrow-\infty
$$

which allows to identify the so far unspecified Cauchy value of the dynamic instanton with the static one: $\varphi_{d}(T, \mathbf{x})=\varphi_{f}(\mathbf{x})=\varphi_{s t}(\mathbf{x})$.

Substitution of the solution $\varphi_{d}$ and $\varphi_{d}^{\prime}$ determined by equations(16), (17) and (18) in dynamic action (4) leads to the remarkable result that the dynamic action on the dynamic instanton solution asymptotically coincides with the static action on the static instanton:

$$
\bar{S}\left(\varphi_{d}, \varphi_{d}^{\prime}\right)=S\left(\varphi_{s t}\right)-S\left(\varphi_{0}\right) \underset{t_{0} \rightarrow-\infty}{\longrightarrow} S\left(\varphi_{s t}\right)
$$

Thus, we arrive at the conclusion that the exponential factor in our steepest descent analysis of correlation function (12) as well as pre-exponential factor asymptotically are the same as in the corresponding equilibrium static theory, the difference being generated by the (fixed, no averaging assumed) initial condition for the field $\varphi_{0}$ only.

Let us explain the choice of the auxiliary equation (16). To consider the asymptotic behaviour of the instanton $\varphi_{d}$ at $t \rightarrow \pm \infty$ we have to study dynamic properties of this equation by investigation of its fixed points. Apart from 
the assumed solution $\varphi_{d}$ discussed so far, equation (16) has two rather obvious time-independent solutions: the trivial solution $\varphi=0$ and the static instanton $\varphi_{s t}$ which obeys $\partial_{t} \varphi_{s t}=0$ and $(\delta S / \delta \varphi)_{\varphi \rightarrow \varphi_{s t}}=0$. The trivial solution does not conform to the non-vanishing initial condition, but gives rise to $\Gamma \delta^{2} S / \delta \varphi^{2}=\Gamma K$. This is a positive definite operator which renders the trivial solution stable in the stationary limit $t_{0} \rightarrow-\infty$. The second variation of the dynamic action for the stationary instanton is $\Gamma\left(K-\frac{|g|}{2} \varphi_{s t}^{2}\right)$. This operator is well known from the instanton analysis of the usual $\varphi^{4}$ model [1]. It has at least one negative eigenvalue which determines the direction along which the solution approaches the stationary instanton at $t \rightarrow \infty$. Such a situation with the time-independent solutions is a heavy argument in favor of the existence of the dynamic instanton which behaves as

$$
\lim _{t \rightarrow t_{0}} \varphi_{d}=\varphi_{0} \underset{t_{0} \rightarrow-\infty}{\longrightarrow} 0, \quad \lim _{t \rightarrow \infty} \varphi_{d}=\varphi_{s t},
$$

even when the explicit solution is not known.

As an example of this statement, consider a simple dynamic model, viz. the zero-dimensional $\varphi^{4}$ model. The dynamic action corresponding to Eq. (1) for the "field" $\varphi(t)$ (without $\mathbf{x}$ dependence) is $S=\frac{\tau}{2} \varphi^{2}+\frac{g}{4 !} \varphi^{4}$. The equilibrium limit of such a model is the famous one-dimensional integral

$$
\int_{-\infty}^{\infty} d x \exp \left(-\frac{\tau}{2} x^{2}-\frac{g}{4 !} x^{4}\right)
$$

which is often considered as an example of applicability of the instanton approach to the large-order asymptotic investigations $[1,2]$. Then equation (16) assumes the form

with the solution

$$
\partial_{t} \varphi-\Gamma\left(\tau \varphi+\frac{g}{6} \varphi^{3}\right)=0
$$

$$
\varphi_{d}=\varphi_{0} \sqrt{\frac{6 \tau}{\left(6 \tau+g \varphi_{0}^{2}\right) \exp \left[-2 \Gamma \tau\left(t-t_{0}\right)\right]-g \varphi_{0}^{2}}} .
$$

This dynamic instanton tends to $\varphi_{s t}=\sqrt{6 \tau /(-g)}$ at $t \rightarrow \infty$ and vanishes at $t \rightarrow-\infty$.

As to the usual $\varphi^{4}$ model in $4-\epsilon$ dimension, we have not been able to find a closed-form representation for the dynamic instanton, but a convergent iterative solution of equation (16) may be constructed as follows. As the zerothorder approximation, take

$$
\varphi_{d}^{(0)}(t, \mathbf{x})=\int d \mathbf{x}^{\prime} \frac{\exp \left[-\frac{\left(\mathbf{x}-\mathbf{x}^{\prime}\right)^{2}}{4 \Gamma(T-t)}\right]}{[4 \pi \Gamma(T-t)]^{d / 2}} \varphi_{s t}\left(\mathbf{x}^{\prime}\right),
$$


which obviously is a solution of the linearized equation with the final condition $\varphi_{d}^{(0)}(T, \mathbf{x})=\varphi_{s t}(\mathbf{x})$. Rewriting equation (16) as the integral equation

$$
\varphi_{d}(t, \mathbf{x})=-\frac{\Gamma g}{6} \int_{t}^{T} d t^{\prime} \int d \mathbf{x}^{\prime} \frac{\exp \left[-\frac{\left(\mathbf{x}-\mathbf{x}^{\prime}\right)^{2}}{4 \Gamma\left(t^{\prime}-t\right)}\right]}{\left[4 \pi \Gamma\left(t^{\prime}-t\right)\right]^{d / 2}} \varphi_{d}^{3}\left(t^{\prime}, \mathbf{x}^{\prime}\right)+\varphi_{d}^{(0)}(t, \mathbf{x})
$$

we immediately see that the next-to-leading term of the iterative sequence is

$$
\varphi_{d}^{(1)}(t, \mathbf{x})=-\frac{\Gamma g}{6} \int_{t}^{T} d t^{\prime} \int d \mathbf{x}^{\prime} \frac{\exp \left[-\frac{\left(\mathbf{x}-\mathbf{x}^{\prime}\right)^{2}}{4 \Gamma\left(t^{\prime}-t\right)}\right]}{\left[4 \pi \Gamma\left(t^{\prime}-t\right)\right]^{d / 2}}\left[\varphi_{d}^{(0)}\left(t^{\prime}, \mathbf{x}^{\prime}\right)\right]^{3}
$$

with the vanishing final value: $\varphi_{d}^{(1)}(T, \mathbf{x})=0$. Proceeding in the same fashion we arrive at the usual tree-graph solution of the nonlinear equation (16) with the given final Cauchy condition instead of the initial condition.

Similar solutions were used in $[13,14]$ for the massive $\varphi^{4}$ model in dimension $4-\epsilon$ in the static limit. There the tree-graph expansion was sufficient to determine the asymptotic behaviour of the instanton at both large and small values of the coordinate argument $|\mathbf{x}|$, which were of practical interest.

It should also be noted that - unlike the usual field-theoretic perturbation expansion - the number of the graphs at large order of the tree expansion has no factorial growth. Therefore, this expansion is convergent in a disk of finite radius and can be analytically continued. For illustration of this statement, consider the expansion in the parameter $g$ of the exact solution of the toy model (19), which obviously has a finite radius of convergence.

Usually we are interested in the logarithmic theory. In this case the model is translation invariant with respect to $\mathbf{x}$ and scale invariant with respect to a synchronous dilatation of $\mathbf{x}$ and $t$ (the scaling dimension of $t$ being twice the scaling dimension of $\mathbf{x}$ ). Therefore, the instanton has an arbitrariness of the form

$$
\varphi_{d}(\mathbf{x}, t)=\frac{1}{y^{D / 2-1}} f_{d}\left(\frac{\mathbf{x}-\mathbf{x}_{0}}{y}, \Gamma \frac{t}{y^{2}}\right),
$$

where $D$ is the space dimension, $\mathbf{x}_{0}$ and $y$ are arbitrary parameters. The coefficient $\Gamma$ in $(20)$ is introduced to make the second argument of $f_{d}$ dimensionless. The translation invariance in space and dilatation invariance forces to lift the corresponding degeneracy in the instanton solution. Due to the non-vanishing value $\varphi_{d}^{\prime}(T)=\left.2 D^{-1} \partial_{t} \varphi_{d}\right|_{t=T}$ of the auxiliary field of the dynamic instanton solution, a shift of the integration space from the original perturbative integration space $(10)$ with $\varphi^{\prime}(T)=0$ to a space with $\varphi^{\prime}(T) \neq 0$ is implied in the functional integrals of the $k$-point correlation functions (12). 
In this integration space the degeneracies of the instanton may be removed with the aid of the same unit decomposition

$$
\begin{aligned}
1=\int d^{D} \mathbf{x}_{0} & \int_{-\infty}^{+\infty} d \ln y^{2} \delta\left[-\frac{g}{24} \int d \mathbf{x} \varphi_{\infty}^{4}(\mathbf{x}) \ln \left(\frac{\mathbf{x}-\mathbf{x}_{0}}{y}\right)^{2}\right] \\
& \times \delta^{D}\left[-\frac{g}{24} \int d \mathbf{x} \varphi_{\infty}^{4}(\mathbf{x})\left(\mathbf{x}-\mathbf{x}_{0}\right)\right]\left[-\frac{g}{24} \int d \mathbf{x} \varphi_{\infty}^{4}(\mathbf{x})\right]^{D+1}
\end{aligned}
$$

as in the static instanton theory, which then imposes conditions on the final value of the integration field: $\varphi(T, \mathbf{x})=\varphi_{\infty}(\mathbf{x})$. \{Relation (21) is written for a scalar field $\varphi$, in a vector case one more contribution due to the rotational invariance is necessary $[1,13]\}$. The $\delta$ functions introduced by the unit decomposition (21) fix the arbitrariness of the instanton completely.

An appropriate change of variables to avoid the spatial translation invariance and dilatation invariance problem is well known $[1,2,13]$. Therefore, we will henceforth consider only specific dynamic properties of the model. All needed operations to deal with the spatial translation invariance and dilatation invariance problems, together with spatial arguments of of fields and spatial integration, are implied and omitted in the following.

Thus, we arrive at the correlation function

$$
\frac{1}{2 \pi i} \oint \frac{d g}{g} \frac{\iint \mathcal{D} \varphi \mathcal{D} \varphi^{\prime} \varphi\left(\mathbf{x}_{\mathbf{1}}, t_{1}\right) \ldots \varphi\left(\mathbf{x}_{\mathbf{k}}, t_{k}\right) \mathrm{I} e^{-\bar{S}-N \lg g}}{\iint \mathcal{D} \varphi \mathcal{D} \varphi^{\prime} e^{-\bar{S}_{0}}}
$$

where I stands for all contributions from the unit decomposition (21). At the leading order in $N$ we replace all $\varphi\left(\mathbf{x}_{\mathbf{i}}, t_{i}\right)$ by $\varphi_{d}$ in the pre-exponential factor. In the stationary limit $t_{0} \rightarrow-\infty$ the initial value of the instanton $\varphi_{d}\left(t_{0}\right)$ vanishes and in the exponential the static instanton action is recovered at leading order in $N$.

\section{Calculation of the fluctuation determinant}

During the calculation of the fluctuation integral the usual stretching of variables $\delta \varphi \rightarrow \delta \varphi / \sqrt{N}, \delta \varphi^{\prime} \rightarrow \delta \varphi^{\prime} / \sqrt{N}$ in the fluctuation integration in the numerator is accompanied by a similar transformation $\varphi \rightarrow \varphi / \sqrt{N}, \varphi^{\prime} \rightarrow \varphi^{\prime} / \sqrt{N}$ in the denominator: the Jacobians thus cancel. We shall not write the factor due to fluctuations of $g$ explicitly, because after the standard change of variables in the original dynamic action $\varphi \rightarrow \varphi / \sqrt{-g}, \varphi^{\prime} \rightarrow \varphi^{\prime} / \sqrt{-g}$ fluctuation contributions due to fields $\delta \varphi$ and $\delta \varphi^{\prime}$ on one hand and due to the coupling 
constant $\delta g$ on the other factorize, the latter giving rise to a trivial numerical factor.

The fluctuation integral over $\delta \varphi$ and $\delta \varphi^{\prime}$ may be written as

$$
\begin{aligned}
\delta \Sigma=\left\{\iint \mathcal{D} \delta \varphi \mathcal{D} \delta \varphi^{\prime} \exp \left[\frac{1}{2} \delta \varphi^{\prime} D \delta \varphi^{\prime}-\delta \varphi^{\prime}\left(\frac{\partial \delta \varphi}{\partial t}+\Gamma K \delta \varphi\right)\right]\right\}^{-1} \\
\times \iint \mathcal{D} \delta \varphi \mathcal{D} \delta \varphi^{\prime} \operatorname{I} \exp \left[\frac{1}{2} \delta \varphi^{\prime} D \delta \varphi^{\prime}-\left.\frac{1}{2} \delta \varphi \Gamma \varphi^{\prime} \frac{\delta^{3} S}{\delta \varphi^{3}}\right|_{\varphi_{d}} \delta\right. \\
\left.-\left.\delta \varphi^{\prime}\left(\frac{\partial}{\partial t}+\Gamma \frac{\delta^{2} S}{\delta \varphi^{2}}\right)\right|_{\varphi_{d}} \delta\right] .
\end{aligned}
$$

For economy of notation, we have used the same symbols for the integration variables $\delta \varphi^{\prime}$ and $\delta \varphi$ in both the denominator and the numerator of (22). On the dynamic instanton we immediately see that

$$
\left.\frac{\delta^{3} S}{\delta \varphi^{3}}\right|_{\varphi_{d}} \Gamma \varphi_{d}^{\prime}=\left.\frac{\delta^{3} S}{\delta \varphi^{3}}\right|_{\varphi_{d}} \frac{\partial \varphi_{d}}{\partial t}=\left.\frac{\partial}{\partial t} \frac{\delta^{2} S}{\delta \varphi^{2}}\right|_{\varphi_{d}}
$$

With the aid of this relation and the change of variables

$$
\delta \varphi^{\prime}=\psi^{\prime}+2 D^{-1} \frac{\partial \delta \varphi}{\partial t}
$$

the quadratic form of the exponential of the numerator of fluctuation integral (22) may be expressed as

$$
\begin{aligned}
-\frac{1}{2} \delta_{\varphi, \varphi^{\prime}}^{2} \bar{S}=\frac{1}{2} \psi^{\prime} D \psi^{\prime}-\psi^{\prime}\left(-\frac{\partial}{\partial t}\right. & \left.+\Gamma K+\Gamma \frac{\delta^{2} S_{I}}{\delta \varphi^{2}}\right)\left.\right|_{\varphi_{d}} \delta \varphi \\
& -\left.\frac{1}{2} \delta \varphi_{s t} \frac{\delta^{2} S}{\delta \varphi^{2}}\right|_{\varphi_{s t}} \delta \varphi_{s t}+\left.\frac{1}{2} \delta \varphi_{0} \frac{\delta^{2} S}{\delta \varphi^{2}}\right|_{\varphi_{0}} \delta \varphi_{0}
\end{aligned}
$$

where $\delta \varphi_{s t}=\left.\delta \varphi\right|_{t=T}$ and $\delta \varphi_{0}=\left.\delta \varphi\right|_{t=t_{0}}$. Note the change of sign of the time derivative in the second term of (24). We also remind that the term $\left.\frac{1}{2} \delta \varphi_{s t} \frac{\delta^{2} S}{\delta \varphi^{2}}\right|_{\varphi_{s t}} \delta \varphi_{s t}$ in (24) describing the effect of asymptotic (equilibrium) fluctuations would be absent in the original perturbative integration space.

We intend to calculate the fluctuation integral over the dynamic fluctuations regarding the term $\left.\psi^{\prime} \Gamma \frac{\delta^{2} S_{I}}{\delta \varphi^{2}}\right|_{\varphi_{d}} \delta \varphi$, where $S_{I}$ is the interaction part of the static action, as perturbation. At leading order in $N$, when only the fluctuation determinant is needed without any other fluctuation contributions, this trick leads to a sum of closed loops of advanced diffusion kernels which essentially reduces the purely dynamic fluctuation determinant to a constant regardless 
of the properties of the full differential operator in (24). The account of dynamic fluctuations becomes highly non-trivial, of course, when pre-exponential fluctuation terms are included.

To arrive at a tractable perturbative expansion of the dynamic fluctuation determinant we write the normalization factor in terms of the same perturbation expansion as indicated in (24). Change of variables (23) casts the free-field action in the denominator in the form

$$
-\frac{1}{2} \delta_{\varphi, \varphi^{\prime}}^{2} \bar{S}_{0}=\frac{1}{2} \psi^{\prime} D \psi^{\prime}-\psi^{\prime}\left(-\frac{\partial}{\partial t}+\Gamma K\right) \delta \varphi-\frac{1}{2} \delta \varphi_{s t} K \delta \varphi_{s t}+\frac{1}{2} \delta \varphi_{0} K \delta \varphi_{0}
$$

with the same free-field part for the perturbative calculation as in (24). A shift of the integration space similar to that in the numerator of the fluctuation integral (22) is implied in (25) as well.

The integration space corresponding to perturbative expansion with (25) as the free-field action includes fields constructed with the aid of the advanced diffusion kernel by the standard prescription, i.e.

$$
\left(\begin{array}{c}
\overline{\delta \varphi} \\
\psi^{\prime}
\end{array}\right)=\left(\begin{array}{cc}
\Delta_{11} & \Delta_{21} \\
\Delta_{12} & 0
\end{array}\right)\left(\begin{array}{l}
\eta \\
\eta^{\prime}
\end{array}\right)=\left(\begin{array}{l}
\Delta_{11} \eta+\Delta_{21} \eta^{\prime} \\
\Delta_{12} \eta
\end{array}\right)
$$

where $\eta$ and $\eta^{\prime}$ are fields vanishing at the boundaries (i.e. at $|\mathbf{x}| \rightarrow \infty, t=T$ and $t=t_{0}$ ). However, the propagator structure leads to final values of the fields $\overline{\delta \varphi}$ and $\psi^{\prime}$, which become arbitrarily small, when $T$ grows. Since in the integration space around the instanton the long-time asymptotics of the fluctuation field $\delta \varphi \rightarrow \delta \varphi_{s t}$ is finite and should eventually be independent of the choice of $T$, an additional contribution is required to account for the asymptotic fluctuations described by $\delta \varphi_{T}$. The spatial convolution of the advanced diffusion kernel with the difference of the desired asymptotic field $\delta \varphi_{s t}$ and the final value of $\overline{\delta \varphi}$ will do:

$$
\delta \varphi_{T}(t, \mathbf{x})=\int d \mathbf{x}^{\prime} \frac{\exp \left[-\frac{\left(\mathbf{x}-\mathbf{x}^{\prime}\right)^{2}}{4 \Gamma(T-t)}\right]}{[4 \pi \Gamma(T-t)]^{d / 2}}\left[\delta \varphi_{s t}\left(\mathbf{x}^{\prime}\right)-\overline{\delta \varphi}\left(T, \mathbf{x}^{\prime}\right)\right]
$$

since it is the solution of the homogeneous equation

$$
\left[-\frac{\partial}{\partial t}+\Gamma\left(-\nabla^{2}\right)\right] \delta \varphi_{T}(t, \mathbf{x})=0
$$

with the final value $\delta \varphi_{T}(T, \mathbf{x})=\delta \varphi_{s t}(\mathbf{x})-\overline{\delta \varphi}(T, \mathbf{x})$.

Due to this, on the finite interval $\left[t_{0}, T\right]$ the free-field action on the integration 
field composed as a sum of (26) and (27), i.e.

$$
\delta \varphi=\overline{\delta \varphi}+\delta \varphi_{T}=\Delta_{11} \eta+\Delta_{21} \eta^{\prime}+\delta \varphi_{T}
$$

amounts to

$$
\begin{aligned}
-\frac{1}{2} \delta_{\varphi, \varphi^{\prime}}^{2} \bar{S}_{0} & =\frac{1}{2} \psi^{\prime} D \psi^{\prime}-\psi^{\prime}\left(-\frac{\partial}{\partial t}+\Gamma K\right) \overline{\delta \varphi} \\
- & \frac{1}{2} \delta \varphi_{T}(T) K \delta \varphi_{T}(T)+\frac{1}{2}\left[\overline{\delta \varphi}_{0}+\delta \varphi_{T}\left(t_{0}\right)\right] K\left[{\overline{\delta \varphi_{0}}}+\delta \varphi_{T}\left(t_{0}\right)\right] .
\end{aligned}
$$

Here, however, $\lim _{T \rightarrow \infty} \delta \varphi_{T}(T)=\delta \varphi_{\text {st }}$ by construction (27). Therefore in (29) the explicit dependence (apart from the upper limit of all time integrals) on $T$ remains only in the initial value of the field $\delta \varphi_{T}\left(t_{0}\right)$, which is finite. Moreover, from (27) it also follows that $\delta \varphi_{T}\left(t_{0}\right) \rightarrow 0$, when $T \rightarrow \infty$. Thus, we may write the original quadratic form on the ray $\left[t_{0}, \infty\right)$ as

$$
\begin{aligned}
-\frac{1}{2} \delta_{\varphi, \varphi^{\prime}}^{2} \bar{S}_{0}=\lim _{T \rightarrow \infty}\left\{\frac{1}{2} \psi^{\prime} D \psi^{\prime}-\psi^{\prime}\left(-\frac{\partial}{\partial t}+\right.\right. & \Gamma K) \overline{\delta \varphi} \\
& \left.-\frac{1}{2} \delta \varphi_{s t} K \delta \varphi_{s t}+\frac{1}{2}{\overline{\delta \varphi_{0}}}_{\bar{\delta}}{\overline{\delta \varphi_{0}}}\right\}
\end{aligned}
$$

- an expression which in the functional integral of the denominator of $(22)$ allows to factorize the asymptotic fluctuations described by $\delta \varphi_{s t}$ from the "purely dynamic" fluctuations described by $\overline{\delta \varphi}$ and $\psi^{\prime}$ from (26).

A similar representation in the quadratic form of fluctuations (24) leaves explicit dependence on $\delta \varphi_{T}$ also in the "interaction" term:

$$
\begin{aligned}
-\frac{1}{2} \delta_{\varphi, \varphi^{\prime}}^{2} \bar{S}=\lim _{T \rightarrow \infty}\{ & \frac{1}{2} \psi^{\prime} D \psi^{\prime}-\psi^{\prime}\left(-\frac{\partial}{\partial t}+\Gamma K\right) \overline{\delta \varphi}-\left.\psi^{\prime}\left(\Gamma \frac{\delta^{2} S_{I}}{\delta \varphi^{2}}\right)\right|_{\varphi_{d}}\left(\overline{\delta \varphi}+\delta \varphi_{T}\right) \\
& \left.-\left.\frac{1}{2} \delta \varphi_{T}(T) \frac{\delta^{2} S}{\delta \varphi^{2}}\right|_{\varphi_{s t}} \delta \varphi_{T}(T)+\left.\frac{1}{2} \delta \varphi_{T}\left(t_{0}\right) \frac{\delta^{2} S}{\delta \varphi^{2}}\right|_{\varphi_{0}} \delta \varphi_{T}\left(t_{0}\right)\right\} .
\end{aligned}
$$

Again $\lim _{T \rightarrow \infty} \delta \varphi_{T}(T)=\delta \varphi_{\text {st }}$ and $\lim _{T \rightarrow \infty} \varphi_{T}\left(t_{0}\right)=0$ by construction. Moreover, as seen from $(27)$, the latter limit applies for almost all $t$ as well: $\left.\lim _{T \rightarrow \infty} \varphi_{T}(t, \mathbf{x})\right)=$ $0, t_{0} \leq t<T$. From this it follows that

$$
\left.\psi^{\prime}\left(\Gamma \frac{\delta^{2} S_{I}}{\delta \varphi^{2}}\right)\right|_{\varphi_{d}} \delta \varphi_{T}=\left.\int_{t_{0}}^{T} \psi^{\prime}(t)\left(\Gamma \frac{\delta^{2} S_{I}}{\delta \varphi^{2}}\right)\right|_{\varphi_{d}} \delta \varphi_{T}(t) d t \underset{T \rightarrow \infty}{\longrightarrow} 0
$$

Therefore, we see that representation (27) and (28) leads to factorization of the time-independent asymptotic fluctuations $\delta \varphi_{s t}$ and the dynamic fluctuations $\overline{\delta \varphi}$ and $\psi^{\prime}$ in the numerator of $(22)$ as well. 
Therefore, the fluctuation integral may be expressed in the explicitly factorized form

$$
\begin{gathered}
\delta \Sigma=\left[\int \mathcal{D} \delta \varphi_{s t} \exp \left(-\frac{1}{2} \delta \varphi_{s t} K \delta \varphi_{s t}\right)\right]^{-1} \int \mathcal{D} \delta \varphi_{s t} \mathrm{I} \exp \left(-\left.\frac{1}{2} \delta \varphi_{s t} \frac{\delta^{2} S}{\delta \varphi^{2}}\right|_{\varphi_{s t}} \delta \varphi_{s t}\right) \\
\left\{\iint \mathcal{D} \overline{\delta \varphi} \mathcal{D} \psi^{\prime} \exp \left[\frac{1}{2} \psi^{\prime} D \psi^{\prime}-\psi^{\prime}\left(-\frac{\partial \overline{\delta \varphi}}{\partial t}+\Gamma K \overline{\delta \varphi}\right)+\frac{1}{2} \overline{\delta \varphi_{0}} K \overline{\delta \varphi_{0}}\right]\right\}^{-1} \\
\times \iint \mathcal{D} \overline{\delta \varphi} \mathcal{D} \psi^{\prime} \exp \left[\frac{1}{2} \psi^{\prime} D \psi^{\prime}-\psi^{\prime}\left(-\frac{\partial}{\partial t}+\Gamma K\right) \overline{\delta \varphi}\right. \\
\left.-\psi^{\prime}\left(\Gamma \frac{\delta^{2} S_{I}}{\delta \varphi^{2}}\right)\left|\frac{1}{\delta \varphi}+\frac{1}{2} \overline{\delta \varphi_{0}} \frac{\delta^{2} S}{\delta \varphi^{2}}\right|_{\varphi_{0}} \overline{\delta \varphi_{0}}\right],
\end{gathered}
$$

with the dynamic fields of the structure (26). On the right-hand-side of (30) the first line yields the familiar static fluctuation determinant including the unit decomposition (21).

A non-trivial dependence on the initial time instant $t_{0}$ remains in (30) through the non-zero initial value of the fluctuation field ${\overline{\delta \varphi_{0}}}_{0}$. This is an integral of the independent variables $\eta$ and $\eta^{\prime}$ with vanishing boundary conditions from representation (26):

$$
\overline{\delta \varphi}_{0}(\mathbf{x})=\int_{t_{0}}^{\infty} d t \int d \mathbf{x}^{\prime}\left[\Delta_{11}\left(t_{0}, t, \mathbf{x}-\mathbf{x}^{\prime}\right) \eta\left(t, \mathbf{x}^{\prime}\right)+\Delta_{21}\left(t_{0}-t, \mathbf{x}-\mathbf{x}^{\prime}\right) \eta^{\prime}\left(t, \mathbf{x}^{\prime}\right)\right]
$$

and thus leads to fairly complicated propagator structure of the perturbative expansion of the dynamic contribution in (30). This also means that a functional dependence on the dynamic instanton remains in the fluctuation determinant for finite $t_{0}$. In the asymptotic limit $t_{0} \rightarrow-\infty-$ which restores the translation invariance with respect to time - the dependence on the instanton vanishes, however. This takes place, because due to the properties of the diffusion kernel, in the limit $t_{0} \rightarrow-\infty$ the initial value of the fluctuation field vanishes: $\overline{\delta \varphi}_{0} \rightarrow 0$. Therefore, in this limit the dynamic part of (30) may be expressed in a form similar to $(7)$ :

$$
\begin{aligned}
\left\{\iint \mathcal{D} \overline{\delta \varphi} \mathcal{D} \psi^{\prime} \exp \left[\frac{1}{2} \psi^{\prime} D \psi^{\prime}-\psi^{\prime}\left(-\frac{\partial \overline{\delta \varphi}}{\partial t}+\Gamma K \overline{\delta \varphi}\right)\right]\right\}^{-1} \\
\times \iint \mathcal{D} \overline{\delta \varphi} \mathcal{D} \psi^{\prime} \operatorname{I} \exp \left[\frac{1}{2} \psi^{\prime} D \psi^{\prime}-\psi^{\prime}\left(-\frac{\partial}{\partial t}+\Gamma K\right) \overline{\delta \varphi}-\psi^{\prime}\left(\Gamma \frac{\delta^{2} S_{I}}{\delta \varphi^{2}}\right)|\overline{\delta \varphi}|_{\varphi_{d}}\right] \\
=\left.\exp \left[\frac{1}{2} \frac{\delta}{\delta \Psi} \Delta^{T} \frac{\delta}{\delta \Psi}\right] \exp \left[-\psi^{\prime}\left(\Gamma \frac{\delta^{2} S_{I}}{\delta \varphi^{2}}\right) \mid \begin{array}{c}
\psi \\
\varphi_{d}
\end{array}\right]\right|_{\Psi=0} \cdot
\end{aligned}
$$

Here $\Delta^{T}$ is obtained from the propagator kernel (8) by replacing the retarded 
diffusion kernel by the retarded one and vice versa. Inspection reveals that the perturbation expansion of (31) contains only closed loops of the advanced propagator which all vanish (including the closed single loop, due to our definition of the $T$ product at coinciding times). Therefore, we immediately see that perturbatively the expression in (31) is equal to unity and the dynamic fluctuation determinant (22) is equal to the static one in the limit $t_{0} \rightarrow-\infty$ :

$$
\lim _{t_{0} \rightarrow-\infty} \delta \Sigma=\frac{\int \mathcal{D} \delta \varphi_{s t} \mathrm{I} \exp \left(-\left.\frac{1}{2} \delta \varphi_{s t} \frac{\delta^{2} S}{\delta \varphi^{2}}\right|_{\varphi_{s t}} \delta \varphi_{s t}\right)}{\int \mathcal{D} \delta \varphi_{s t} \exp \left(-\frac{1}{2} \delta \varphi_{s t} K \delta \varphi_{s t}\right)}
$$

Thus, not only the pre-exponential and exponential factors in the large $N$ asymptote of (12) coincide with the static expression, but the fluctuation integrals with the chosen regularization of determinants coincide as well.

\section{Dynamic part of the correlation function and response function}

We have shown that dynamic correlation functions coincide with the static ones at leading order in $1 / N$ in the limit $t_{0} \rightarrow-\infty$, when the model is translational invariant in time. Leading-order contributions to response functions vanish in this case, however. To see this, consider the $N$ th-order contribution to the response function

$$
\frac{1}{2 \pi i} \oint \frac{d g}{g} \frac{\iint \mathcal{D} \varphi \mathcal{D} \varphi^{\prime} \varphi\left(\mathbf{x}_{1}, t_{1}\right) \varphi^{\prime}\left(\mathbf{x}_{2}, t_{2}\right) \mathrm{I} e^{-\bar{S}-N \lg g}}{\iint \mathcal{D} \varphi \mathcal{D} \varphi^{\prime} e^{-\bar{S}_{0}}}
$$

On the instanton solution (16) and (17) the pre-exponential factor in (33) includes the factor

$$
\varphi_{d}^{\prime}\left(\mathbf{x}_{2}, t_{2}\right)=2 D^{-1} \frac{\partial}{\partial t_{2}} \varphi_{d}\left(\mathbf{x}_{2}, t_{2}\right)
$$

which vanishes in the limit $t_{0} \rightarrow-\infty$ due to the necessary asymptotic properties of the instanton, which approaches a finite time-independent limit at long times after the initial time instant. Thus, the leading contribution to the response function is given by corrections of order $1 / N$.

To capture dynamic effects it is sufficient to consider only corrections effected by the replacement $\varphi \rightarrow \delta \varphi$ and $\varphi^{\prime} \rightarrow \delta \varphi^{\prime} .1 / N$ corrections of any other type lead only to the small changes to the stationary results obtained previously (without any dynamic dependence). 
The fluctuation integral for the pair correlation function is

$$
\begin{aligned}
& \delta \Sigma_{\varphi \varphi}=\left\{\iint \mathcal{D} \delta \varphi \mathcal{D} \delta \varphi^{\prime} \exp \left[\frac{1}{2} \delta \varphi^{\prime} D \delta \varphi^{\prime}-\delta \varphi^{\prime}\left(\frac{\partial \delta \varphi}{\partial t}+\Gamma K \delta \varphi\right)\right]\right\}^{-1} \\
& \times \iint \mathcal{D} \delta \varphi \mathcal{D} \delta \varphi^{\prime} \delta \varphi\left(\mathbf{x}_{1}, t_{1}\right) \delta \varphi\left(\mathbf{x}_{2}, t_{2}\right) \operatorname{I} \exp \left[\frac{1}{2} \delta \varphi^{\prime} D \delta \varphi^{\prime}-\left.\frac{1}{2} \delta \varphi \Gamma \varphi_{d}^{\prime} \frac{\delta^{3} S}{\delta \varphi^{3}}\right|_{\varphi_{d}} \delta \varphi\right. \\
& \left.-\left.\delta \varphi^{\prime}\left(\frac{\partial}{\partial t}+\Gamma \frac{\delta^{2} S}{\delta \varphi^{2}}\right)\right|_{\varphi_{d}} \delta \varphi\right]
\end{aligned}
$$

With the use of the change of variables (23) and the subsequent decoupling of dynamic and static fluctuations according to (26), (27) and (28), this fluctuation integral gives rise to the sum

$$
\begin{gathered}
\delta \Sigma_{\varphi \varphi}=\frac{\int \mathcal{D} \delta \varphi_{s t} \delta \varphi_{s t}\left(\mathbf{x}_{1}\right) \delta \varphi_{s t}\left(\mathbf{x}_{2}\right) \mathrm{I} \exp \left(-\left.\frac{1}{2} \delta \varphi_{s t} \frac{\delta^{2} S}{\delta \varphi^{2}}\right|_{\varphi_{s t}} \delta \varphi_{s t}\right)}{\int \mathcal{D} \delta \varphi_{s t} \exp \left(-\frac{1}{2} \delta \varphi_{s t} K \delta \varphi_{s t}\right)} \\
+\left[\int \mathcal{D} \delta \varphi_{s t} \exp \left(-\frac{1}{2} \delta \varphi_{s t} K \delta \varphi_{s t}\right)\right]^{-1} \int \mathcal{D} \delta \varphi_{s t} \mathrm{I} \exp \left(-\left.\frac{1}{2} \delta \varphi_{s t} \frac{\delta^{2} S}{\delta \varphi^{2}}\right|_{\varphi_{s t}} \delta \varphi_{s t}\right) \\
\quad\left\{\iint \mathcal{D} \overline{\delta \varphi} \mathcal{D} \psi^{\prime} \exp \left[\frac{1}{2} \psi^{\prime} D \psi^{\prime}-\psi^{\prime}\left(-\frac{\partial \overline{\delta \varphi}}{\partial t}+\Gamma K \overline{\delta \varphi}\right)+\frac{1}{2} \overline{\delta \varphi_{0}} K \overline{\delta \varphi_{0}}\right]\right\}^{-1} \\
\left.\quad-\psi^{\prime}\left(\Gamma \frac{\delta^{2} S_{I}}{\delta \varphi^{2}}\right)\left|\frac{\partial}{\delta \varphi}+\frac{1}{2} \bar{\delta} \psi_{\varphi_{d}} \bar{\delta} \frac{\delta^{2} S}{\delta \varphi^{2}}\right|_{\varphi_{0}} \bar{x}_{1}, t_{1}\right) \overline{\delta \varphi}\left(\mathbf{x}_{2}, t_{2}\right) \exp \left[\frac{1}{2} \psi^{\prime} D \psi^{\prime}-\psi^{\prime}\left(-\frac{\partial}{\partial t}+\Gamma K \overline{\delta \varphi}\right.\right.
\end{gathered}
$$

where the first term is the static fluctuation correlation function and in the second term the dynamic part - multiplied by the static fluctuation determinant (32) - may be expressed formally as

$$
\Delta_{12}\left[1+\left.\Gamma \frac{\delta^{2} S_{I}}{\delta \varphi^{2}}\right|_{\varphi_{d}} \Delta_{12}\right]^{-1} D\left[1+\left.\Delta_{21} \Gamma \frac{\delta^{2} S_{I}}{\delta \varphi^{2}}\right|_{\varphi_{d}}\right]^{-1} \Delta_{21}
$$

in the limit $t_{0} \rightarrow-\infty$.

\section{Conclusion}

In this paper first steps have been taken towards large-order $(N \rightarrow \infty)$ asymptotic analysis of non-linear stochastic field theories with the aid of the instan- 
ton method. It is shown that this approach can be used in dynamic models as well as in the static ones.

For the near-equilibrium model A we have shown that in the temporal translationinvariant case asymptotic properties at leading order in $N$ are almost completely determined by the corresponding static instanton solution and fluctuations thereabout which leads to factorial behaviour of the large-order contributions similar to that in the static instanton analysis: viz. the large-order asymptote for an arbitrary quantity $F$ behaves as

$$
F^{[N]}=N ! C a^{N} N^{b}
$$

where $F^{[N]}$ is the $N$ th order of the expansion of $F$ in the parameter $e(e$ is the coupling constant $g$ or the dimensional regularization parameter $\epsilon$ ), $a$ and $b$ are constants known from the analysis of the static theory $[1,2], C$ is an amplitude factor - either a constant or a function of coordinate and time arguments depending on the quantity $F$. In the static $\varphi^{4}$ model the functions $C$ for universal quantities have been calculated in Ref. [14].

In our treatment it has been quite essential that the dynamic stochastic problem has a Gibbsian limit, which physically is not totally unexpected due to the fluctuation-dissipation theorem.

Having been interested mainly in proving the factorial form of the large-order asymptotes in the dynamic model considered we have not dwelled on the problem of effects of renormalization. However, in the static instanton analysis - according to $[1,2]$ - the renormalization does non affect the solution of the stationarity equation and at leading order in $1 / N$ it contributes to the $C$-type constants in correlation and response functions only. Details of the correct description of the renormalization in the instanton approach in the minimalsubtraction scheme can be found in Ref. [4]. Similar methods are needed for accurate calculation of the constants in our asymptotic expressions.

As to the calculation of the dynamic critical exponent $z$, the renormalizationgroup equation for, e.g., the renormalized two-point function $W_{2}=\langle\varphi \varphi\rangle^{R}$ at the fixed point $g_{*}$

$$
\left(k \frac{\partial}{\partial k}-z t \frac{\partial}{\partial t}+\Delta\right) W_{2}=0
$$

may be used. In equation (35), $k$ is the wave number and $\Delta$ is the scaling dimension known from the static theory. Details of the transformation of the $g$ expansion considered above into the $\epsilon$ expansion are described in Refs. $[1,2]$. From the factorial form of large orders in the $\epsilon$ expansion the asymptotic estimate for the expansion of the exponent $z$ follows:

$$
z^{[N]} \sim \frac{k \partial_{k} W_{2}^{[N]}+\Delta^{[0]} W_{2}^{[N]}+\Delta^{[N]} W_{2}^{[0]}-z^{[0]} t \partial_{t} W_{2}^{[N]}+X^{[N]}}{t \partial_{t} W_{2}^{[0]}}
$$


where $X^{[N]}$ stands for corrections in $1 / N$. Expression (36) demonstrates that the value $z^{[N]}$ has the same factorial form (34) as the static critical exponents.

\section{References}

[1] J. Zinn-Justin, Quantum Field Theory and Critical Phenomena, 3rd edition (Clarendon Press, Oxford, 1996).

[2] L.N. Lipatov, Zh. Eksp. Teor. Fiz. 72 (1977) 411.

[3] E.Brezin, J.C. Le Guillou and J. Zinn-Justin, Phys. Rev. D 15 (1977) 1544.

[4] M.V. Komarova and M.Yu. Nalimov, Theor. Math. Phys. 126 (2001) 393.

[5] P.C. Martin, E.D. Siggia and H.A. Rose, Phys. Rev. A 8 (1973) 423.

[6] M. Chertkov, Phys. Rev. E 55 (1997) 2722.

[7] E. Balkovsky and V. Lebedev, Phys. Rev. E 58 (1998) 5776.

[8] G. Falkovich, K. Gawędzki and M. Vergassola, Rev. Mod. Phys. 73 (2001) 913.

[9] P.C. Hohenberg and B.I. Halperin, Rev. Mod. Phys. 49 (1977) 435.

[10] C. De Dominicis, J. Phys. (Paris) 37, Suppl C1 (1976) 247;

H.K. Janssen, Z. Phys. B 23 (1976) 377.

[11] A.N. Vasiliev, Functional Methods in Quantum Field Theory and Statistical Physics (Gordon and Breach, Amsterdam, 1998).

[12] K. Symanzik, Nucl. Phys. B 190 (1981) 1.

[13] L.M. Suslov, JETP 84 (1997) 120.

[14] M.V. Komarova and M.Yu. Nalimov, Theor. Math. Phys. 129 (2001) 387. 